\title{
CLINICAL AND RADIOGRAPHIC OUTCOMES OF RADICULAR CYST ENUCLEATION USING PIEZOSURGERY VERSUS CONVENTIONAL SURGERY
}

\author{
Marwa G. Noureldin* and Lydia N. Melek**
}

\begin{abstract}
AIM: The study was intended to evaluate the effectiveness of piezosurgery in enucleation of radicular odontogenic cysts in comparison to the conventional technique from both the clinical and radiographic perspectives.

Methods: Fourteen ( 8 females and 6 males) patients with age range of 30-55 years who had radicular cysts associated with non-vital teeth, were operated at the Oral and Maxillofacial Surgery Department, Faculty of Dentistry, Alexandria University. The patients were randomly divided into 2 groups;1) the study group (7 patients) in which cyst enucleation using piezosurgery was employed, 2) control group in which the cysts were removed using the conventional technique. The following parameters were assessed; postoperative pain, time of operation, hemorrhage control and field visibility, ease of operation, neurosensory evaluation and radiographic percentage of change in bone defect volume between preoperative and 3-months postoperative values.
\end{abstract}

Results: 9 maxillary and 5 mandibular radicular cysts were enucleated in both groups. The piezosurgery group showed a statistically significant control over heamorrhage and a clearer, more visible field when compared to the conventional group. The difference between the times required for cyst enucleation in both groups was statistically significant, with longer time recorded in the piezosurgery group. No complications were observed during execution of piezosurgery techniques. On the other hand, with the conventional technique, 2 patients complained of neurosensory involvement which was resolved over time. Also, the piezosurgery group showed a significantly more reduction of bone defect volume than the conventional surgery group, 3 months after the operation in comparison to the preoperative baseline value.

Conclusions: Piezosurgery proved to be more effective for cyst enucleation than the conventional method. Moreover, the piezosurgery was a more efficient choice for preservation of the important anatomical structures while providing a clearer field of surgery. Also, better early healing potential of the bone defect resulting from cyst enucleation was detected with piezosurgery.

KEYWORDS: Piezosurgery, cyst enucleation, radicular cyst, periapical cyst

* Lecturer of Oral and Maxillofacial Surgery, Alexandria University

** Assistant Professor of Oral and Maxillofacial Surgery, Alexandria University 


\section{INTRODUCTION}

Common lesions of the oral cavity include cysts particularly radicular cysts. Enucleation is one of the treatment modalities which involves complete removal of the cystic lining in addition to primary closure of the resultant cavity. ${ }^{(1)}$ This procedure alone or combined with others, is considered to be the treatment of choice, since the entire pathological lesion is removed. ${ }^{(2)}$

Conventional tools such as burs, oscillating saws, rongeurs and curettes are aggressive instruments that do not target the bone only. The vital anatomical structures related to the region of surgery such as the maxillary sinus in the maxilla and the neurovascular bundle in the mandible are liable to be injured using these traditional instruments. Moreover, these tools can lead to tearing of the cystic membrane leading to incomplete removal of the lesion leaving behind fragmented pathological tissues which can be the seed for recurrence. ${ }^{(3)}$

Since piezosurgery has been introduced to the field of maxillofacial surgery, it has proven to have promising results. It targets the bone during cutting, safely preserving the soft tissue. (4) Moreover, it reduces post-operative bleeding resulting in better visibility during the surgical operation. ${ }^{(5)}$ Numerous authors reported that piezosurgery preserves the sinus walls thus reduces the chance of sinus perforation. This advantage would be helpful in cyst enucleation, since the preservation of the cystic lining is essential for successful treatment outcome. ${ }^{(6-9)}$

Deficient information was reported in the literature regarding the long term follow up for cyst enucleation using piezosurgery. Therefore, this study aimed to evaluate the efficiency and performance of piezosurgery in cyst enucleation in comparison to conventional techniques.

\section{MATERIALS AND METHODS}

\section{Patients}

This study was conducted on 14 patients ( 8 females and 6 males) with age range of 3055 years who reported to Department of Oral and Maxillofacial surgery, Faculty of Dentistry, Alexandria University, in the year 2019. All patients were requiring enucleation of evident periapical cysts associated with non-vital teeth within the maxilla or mandible. This study followed the Declaration of Helsinki on medical protocol and ethics and the regional Ethical Review Board of the Faculty of Dentistry, Alexandria University approved the study. The trial was also registered on Clinical Trials.gov (ClinicalTrials.gov Identifier: NCT04244422). All participants signed an informed consent agreement.

The patients were randomly divided into two equal groups, a piezosurgery group and a conventional surgery group. The choice of performing either procedure (piezosurgery or conventional surgery) was determined by tossing a coin and the patients were randomly assigned to each group accordingly. The same surgeons executed the enucleation procedure.

All patients were radiographically examined using cone beam computed tomography (CBCT) to evaluate the lesion's size, and its approximation to the anatomical vital structures (maxillary sinus or inferior alveolar neurovascular bundle). (Fig.1)

The inclusion criteria were:

1. Periapical cysts involving more than one tooth in close relation to the maxillary sinus, nasal cavity or inferior alveolar neurovascular bundle,

2. Intact labial/buccal cortical plate with no perforation.

The exclusion criteria were:

1. Patients with systemic diseases contraindicating surgery,

2. Cases in suspicion of malignancy. 
Following the aseptic surgical protocol, all patients were treated under local anaesthesia using Mepicaine-L (Mepivacaine 36mg/1.8ml + Levonordefrine $0.09 \mathrm{mg} / 1.8 \mathrm{ml}$, Alexandria Co. for pharmaceuticals and chemical industries, Alexandria, Egypt). A mucoperiosteal flap was elevated, then bone removal and cyst enucleation were performed using either piezosurgery for group 1 or conventional technique with rotary and hand instruments for group 2.

\section{Conventional surgery with rotary instruments}

A carbide bur was used to remove the bone to uncover the cyst. A periosteal elevator and a curette were used to aid in the removal of the cystic lesion.

\section{Piezosurgery}

SATLEC ACTEON peizotome 2 (A company of ACTEON Group, France) was used. The appropriate tips were secured to the hand piece and the osteotomy was designed to create a rectangular bony window over the outline of the cyst (Fig.2) which was then carefully elevated and kept in saline. Various tips were used for bone cutting and separating the cystic lining from the surrounding bone. After complete removal of the cyst, the bony window was replaced back in place and the flap was sutured.

In all cases, root canal treatment of the involved teeth was performed prior to surgery. In both groups, the apices of the involved teeth were excised during the surgery.

All specimens were sent for confirmation of final diagnosis by histopathological examination at the department of oral pathology, Faculty of Dentistry, Alexandria University.

All patients were given post-operative instructions and prescribed Augmentin (Amoxycillin+ Clavulanate Potassium, GlaxoSmithKline) 1gm orally every 12 hours daily for 5 days. Also, an antiedematous, anti-inflammatory agent Alphintern (Chymotrypsin 300 E.A.U, Amoun pharmaceutical Co., S.A.E) was prescribed 3 times daily for 5 days.

\section{Parameters assessed:}

1. Postoperative pain level using VAS (0-10) after 24 hours, 48 hours and 1 week, where zero indicates no pain and 10 indicates the maximum pain level.

2. Time of the operation in minutes from start of the incision to the last suture.

3. Hemorrhage control and visibility of the field

4. Ease of operation

5. To assess the visibility of the field and ease of operation, a visual analogue scale (VAS) was used. Each parameter constituted a horizontal $100 \mathrm{~mm}$ continuous line in which value of zero indicates minimum level of the assessed parameter and 100 indicates the maximum level. Each surgeon marked on the line then the score is determined by measuring in mms from the left end of the line to that marked point. ${ }^{10}$

6. Objective assessment of neurosensory function: This was performed using a probe to prick the patient's skin relevant to the area of operation to assess the neurosensory function in comparison to the contralateral side. If the patient states a difference in sensation between the side of operation and the normal side, it was assigned as " $Y$ " and if there was no difference between the two sides, it was assigned as "N".

7. Radiographically: percentage of change in the volume of bone defect between preoperative and 3-months postoperative values was calculated using the CBCT OnDemand3D ${ }^{\mathrm{TM}}$ software (310 Goddard Way, Suite 250 Irvine, CA USA, https://www.ondemand3d.com) to indicate early healing potential. (Fig.3)

\section{Statistical analysis}

Statistical significance was set at $\mathrm{p} \leq 0.05$ and the data obtained from clinical and radiographic findings was analyzed using Statistical Package for Social Sciences SPSS version 21.0 


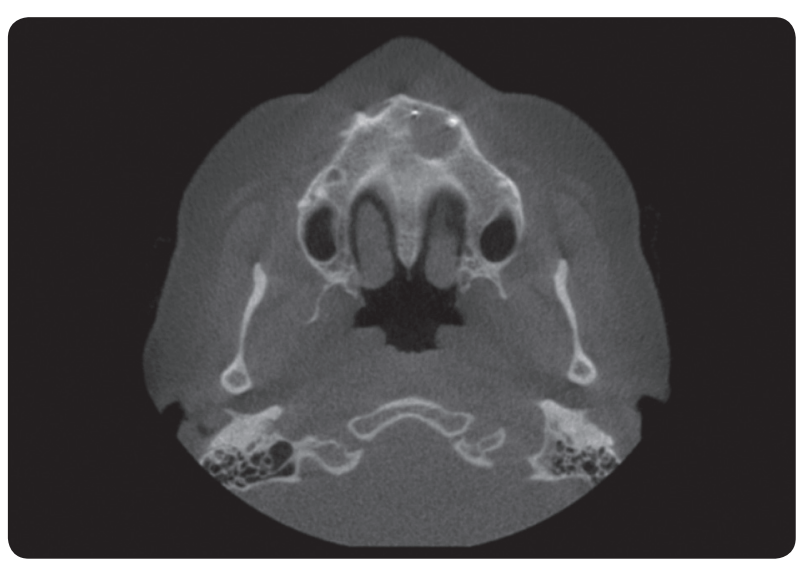

Fig. (1) Preoperative CBCT of Maxillary cyst

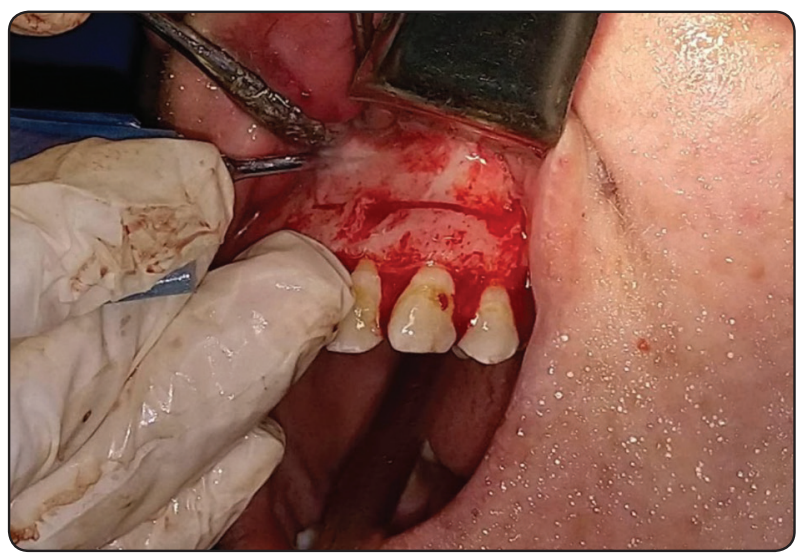

Fig. (2) Cutting of bone window to expose the cyst using piezosurgery

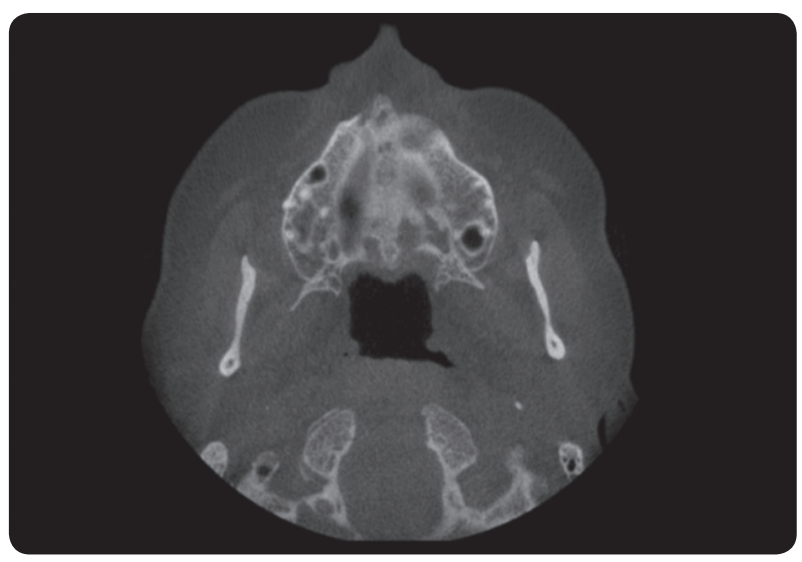

Fig. (3) Postoperative CBCT 3 months after cyst enucleation using piezosurgery

\section{RESULTS}

Fourteen patients ( 8 females and 6 males) were enrolled in this study, with age ranging between 31 and 52 years. The mean age was $40.7 \pm 6.9$ years. The cysts operated included 5 mandibular lesions (35.7\%) and 9 maxillary lesions (64.3\%). Histopathological examination confirmed the diagnosis of inflammatory radicular cysts. The healing was uneventful in all patients without dehiscence or infection.

\section{Pain level}

The mean postoperative pain score for group 1 was lower than that of group 2 after 24 hours, 48 hours and after 1 week, and the difference was statistically significant between the two groups at all time periods. (Table 1)

\section{Operation time}

The mean operation time was $68 \pm 12.45$ minutes in group 1 using piezosurgery, while it was $44.43 \pm 12.74$ minutes in group 2 using the conventional surgery. The difference between the 2 groups was statistically significant. (Table 2)

\section{Visibility of surgical field:}

The mean visibility of surgical field on the 100 mm-VAS for group 1 was $88.57 \pm 3.78$, while it was $79.29 \pm 3.45$ for group 2 . The difference between the two groups was statistically significant. (Table 3 )

\section{Ease of the operation:}

The mean value of operation ease on the $100-\mathrm{mm}$ VAS for the piezosurgery group was $77.86 \pm 4.88$, while for the conventional surgery group it was $73.57 \pm 5.56$. The difference between the two groups was not statistically significant. (Table 4)

\section{Objective assessment of neurosensory function:}

No neurosensory dysfunction was detected in patients of group 1 using the piezosurgery. On the other hand, with the conventional technique, 2 patients complained of neurosensory involvement of the inferior alveolar nerve which was completely resolved over time. The difference was not significant between the two groups. 
Radiographic percentage of change in bone defect volume:

The Mean percentage of change (reduction) in bone defect volume in group 1 was $46.58 \%$, while the mean percentage of change (reduction) in bone defect volume in group 2 was $33.04 \%$. The difference between the 2 groups was statistically significant. (Table 5, Fig. 4)

TABLE (1) Pain level at different time periods in the 2 groups

\begin{tabular}{|c|c|c|c|c|c|c|}
\hline & Group & $\mathrm{N}$ & Mean & Std. Deviation & $\mathrm{t}$ & $\mathrm{p}$ \\
\hline \multirow{2}{*}{ Pain 24 hours } & Group 1 & 7 & 5.9571 & .45774 & \multirow{2}{*}{-8.525} & \multirow{2}{*}{$.000^{*}$} \\
\hline & Group 2 & 7 & 8.0143 & .44508 & & \\
\hline \multirow{2}{*}{ Pain 48 hours } & Group 1 & 7 & 3.8143 & .58146 & \multirow{2}{*}{-9.801} & \multirow{2}{*}{$.000 *$} \\
\hline & Group 2 & 7 & 6.7143 & .52418 & & \\
\hline \multirow{2}{*}{ Pain 1 week } & Group 1 & 7 & 1.2857 & .23401 & \multirow{2}{*}{-3.073} & \multirow{2}{*}{$.010^{*}$} \\
\hline & Group 2 & 7 & 1.6286 & .17995 & & \\
\hline
\end{tabular}

*statistically significant, $p<0.05$

TABLE (2) Comparison between the operation time in the 2 groups

\begin{tabular}{|c|c|c|c|c|c|c|}
\hline & Group & $\mathrm{N}$ & Mean & Std. Deviation & $\mathrm{t}$ & $\mathrm{p}$ \\
\hline \multirow{2}{*}{ Operation time } & Group 1 & 7 & 68.0000 & 12.44990 & 3.501 & $.004^{*}$ \\
\cline { 2 - 7 } & Group 2 & 7 & 44.4286 & 12.73914 & & \\
\hline
\end{tabular}

*statistically significant, $p<0.05$

TABLE (3) Visibility of the surgical field in the 2 groups

\begin{tabular}{|c|c|c|c|c|c|c|}
\hline & Group & $\mathrm{N}$ & Mean & Std. Deviation & $\mathrm{t}$ & $\mathrm{p}$ \\
\hline \multirow{2}{*}{ Visibility of surgical field } & Group 1 & 7 & 88.5714 & 3.77964 & \multirow{2}{*}{4.801} & $.000^{*}$ \\
\cline { 2 - 7 } & Group 2 & 7 & 79.2857 & 3.45033 & \\
\hline
\end{tabular}

*statistically significant, $p<0.05$

TABLE (4) Ease of the operation in the 2 groups

\begin{tabular}{|l|l|r|r|r|r|r|}
\hline & Group & $\mathrm{N}$ & \multicolumn{1}{c|}{ Mean } & \multicolumn{1}{c|}{ Std. Deviation } & $\mathrm{t}$ & $\mathrm{p}$ \\
\hline \multirow{2}{*}{ Ease of operation } & Group 1 & 7 & 77.8571 & 4.87950 & \multirow{2}{*}{1.532} & .151 \\
\cline { 2 - 5 } & Group 2 & 7 & 73.5714 & 5.56349 & & \\
\hline
\end{tabular}


TABLE (5) percentage of change in bone defect volume in the 2 groups

\begin{tabular}{|c|c|c|c|c|c|c|}
\hline & Group & $\mathrm{N}$ & Mean & Std. Deviation & $\mathrm{t}$ & $\mathrm{p}$ \\
\hline \multirow{2}{*}{ Percentage of change } & Group 1 & 7 & 46.5772 & 9.90263 & & \multirow{2}{*}{3.385} \\
\cline { 2 - 7 } & Group 2 & 7 & 33.0372 & 3.73568 & & $.005^{*}$ \\
\hline
\end{tabular}

*statistically significant, $p<0.05$

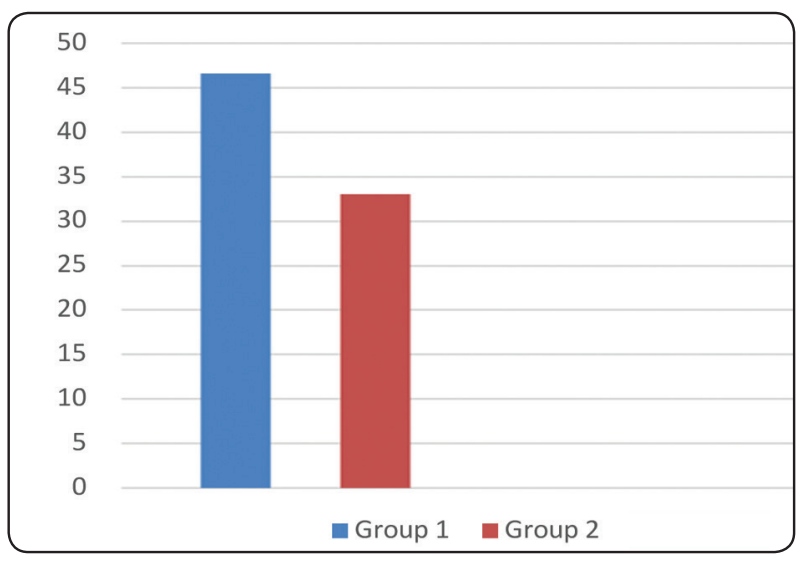

Fig. (4) Mean Percentage of change in bone defect volume within the 2 groups

\section{DISCUSSION}

Piezosurgery is a trendy technology for bone cutting. It is popularly used in various procedures in the field of oral and maxillofacial surgery. ${ }^{(11)}$ Piezosurgery involves the conversion of electric current to ultrasonic frequency vibrations. These vibrations are then concentrated at the tip of the device which results in a cutting effect on mineralized tissues. This cutting effect is known as "cavitation phenomenon". ${ }^{(12)}$

During major surgical procedures, the ability to control and limit hemorrhage becomes a priority. The use of piezosurgery allows an almost bloodless surgical field providing a clearer visibility. The reason is the cavitation effect which is produced by the distribution of cooling fluid of irrigation as well as the vibration generated in the instrument. ${ }^{(13)}$ This corresponds with our study in which piezosurgery- unlike the conventional surgery- provided a field which was visibly clear of blood with the aid of sufficient saline irrigation that continuously washed the blood away. Moreover, the cooling effect of saline prevents over heating of the bone by lowering the temperature as stated by Wallace et al, Landes et al and Yun et al. ${ }^{(6,14,15)}$

Regarding the operating time, our timing showed that piezosurgery was significantly more time consuming than the conventional surgery. This was in agreement with Yaman and Suer, ${ }^{(10)}$ in which the ultrasonic surgery in enucleating cystic lesions of the jaw prolonged the operation time by up to $40 \%$ in comparison to the conventional method. Also, we believe that the operator's experience as well as the learning curve associated with this new surgical practice may have attributed to the prolonged operation time. These findings are supported by Salami et al. ${ }^{(16)}$ as well as Pappalardo and Guarnieri. (17) Moreover, changing the working tips according to the accessibility required for proper osteotomy of the bony window consumed additional time. ${ }^{(10,18)}$

Yaman and Suer ${ }^{(10)}$ also stated that the operating time shouldn't be considered as a fundamental asset for successful enucleation of cystic lesions. This statement agrees with kocigit et al, (4) who concluded that despite the prolonged operating time, piezosurgery proved more success in radicular cysts enucleation than conventional surgery in relation to heamorrhage, perforation of the cystic epithelium as well as the risk of complications. Our results were also in agreement with a recent study by Akcay et al ${ }^{(19)}$ who concluded that piezosurgery 
was a more effective method for cyst enucleation and bone regeneration as well as minimizing postoperative complications. But unlike our results, their study showed that piezosurgery consumed less time and was more cost effective than the traditional techniques.

With respect to the ease of operation, our VAS scores showed more ease with piezosurgery yet the difference between the two techniques was insignificant. The preservation of important anatomical structures as the maxillary sinus membrane in the maxilla and the inferior alveolar neurovascular bundle in the mandible was challenging for complete cystectomy in the conventional surgery group. Two patients complained of postoperative neurosensory involvement which was completely resolved over time. These results agreed with kocigit et al (4) in which no complications were documented in any of the 20 patients treated using piezosurgery, while from the 10 patients treated using conventional methods, 5 cases showed perforation of the cystic epithelium and difficulties in enucleation, and in 2 cases, postoperative hemorrhage occurred.

Although piezosurgery was efficient in bone cutting, manual instruments were also used since difficulty was encountered in some cases where the cystic membrane was tightly adherent to the bony window or near the roots of the related teeth. This was in consensus with Yaman and Suer, (10) who stated that manual instruments provide a more tactile feeling for facilitating the removal of the cystic lining adhering to the thin, delicate anatomical structures.

In reference to our radiographic findings, reduction in bone defect volume was significant with piezosurgery than conventional surgery. This indicates that piezosurgery was found to be more effective than conventional surgery regarding bone healing. Furthermore, piezosurgery provided accurate bone cutting with minimal trauma allowing the bony window to be precisely cut over the outline of the cyst. Consequently, after enucleation, accurate repositioning of the bony window was easily performed. This outcome was in accordance with Ihan Hren and Miljavec, ${ }^{(20)}$ who reported that the outline of the remaining bone defect and the loss of bony wall appear to be the most crucial factors affecting bone healing after cystic enucleation.

Our results showing more accelerated filling of the bone defect in the piezosurgery group, agree with the results of the study by Vercelotti et al (7) who attributed this to the precise, atraumatic cutting that piezoelectric surgery provides. Moreover, other studies comparing the piezosurgery to the conventional surgery have reported better healing, reduced bone loss and less complications associated with piezoelectric surgery. ${ }^{(21,22)}$

To the best of our knowledge, this is the first study comparing the percentage of reduction in bone defect volume after cyst enucleation using piezosurgery versus conventional surgery. The CBCT software enabled the calculation of bone defect volume in the 3 dimensions which gave a more accurate picture of spatial bone healing than just measuring the 2- dimensional surface area done in previous studies. ${ }^{(22)}$

\section{CONCLUSION}

In conclusion, piezosurgery proved to be more effective for cyst enucleation than the conventional method. Moreover, the piezosurgery was a more efficient choice for preservation of the important anatomical structures as well as the cortical plate of bone while providing a clearer field of surgery. Also, better early healing potential of the bone defect resulting from cyst enucleation was detected with piezosurgery. In this study, the results were related to this specific piezosurgical device. Therefore, future studies using other piezosurgery devices are recommended as well as a longer term follow up for cystic lesions. 


\section{REFERENCES}

1. Sabino-Bezerra JR, Santos-Silva AR, Jorge J, Gouvêa AF, Lopes MA: Atypical presentations of simple bone cysts of the mandible: A case series and review of literature. $\mathrm{J}$ Cranio-Maxillofac Surg 41: 391, 2013.

2. Ellis III E. Surgical management of oral pathologic lesions. In: Peterson LJ, Ellis III E, Hupp JR, Tucker MR, editors. Contemporary oral and maxillofacial surgery. $4^{\text {th }}$ ed. St. Louis: Mosby; 2003. p. 481-3.

3. Marx R, Stern D. Oral and maxillofacial pathology: a rationale for diagnosis and treatment. Chicago: Quintessence Publishing; 2003: 573-633.

4. Kocyigit I, Atil F, Alp Y, Tekin U, Tuz H: Piezosurgery Versus Conventional Surgery in Radicular Cyst Enucleation. J Craniofac Surg 23: 1805, 2012.

5. Sakkas N, Otten J-E, Gutwald R, Schmelzeisen R: Transposition of the mental nerve by piezosurgery followed by postoperative neurosensory control: a case report. Br J Oral Maxillofac Surg 46: 270, 2008.

6. Wallace SS, Mazor Z, Froum SJ, Cho S-C, Tarnow DP: Schneiderian membrane perforation rate during sinus elevation using piezosurgery: clinical results of 100 consecutive cases. Int J Periodontics Restorative Dent 27: 413, 2007.

7. Vercellotti T, De Paoli S, Nevins M: The piezoelectric bony window osteotomy and sinus membrane elevation: introduction of a new technique for simplification of the sinus augmentation procedure. Int $\mathrm{J}$ Periodontics Restorative Dent 21: 561, 2001.

8. Stübinger S, Saldamli B, Seitz O, Sader R, Landes CA: Palatal versus vestibular piezoelectric window osteotomy for maxillary sinus elevation: a comparative clinical study of two surgical techniques. Oral Surg Oral Med Oral Pathol Oral Radiol Endod 107: 648, 2009.

9. Seoane J, López-Niño J, García-Caballero L, Seoane-Romero JM, Tomás I, Varela-Centelles P: Membrane perforation in sinus floor elevation - piezoelectric device versus conventional rotary instruments for osteotomy: an experimental study. Clin Implant Dent Relat Res 15: 867, 2013.

10. Yaman Z, Suer BT: Clinical comparison of ultrasonic surgery and conventional surgical techniques for enucleating jaw cysts. Int J Oral Maxillofac Surg 42: 1462, 2013.

11. Pavlíková G, Foltán R, Horká M, Hanzelka T, Borunská H,
Sedý J: Piezosurgery in oral and maxillofacial surgery. Int J Oral Maxillofac Surg 40: 451, 2011.

12. Crosetti E, Battiston B, Succo G: Piezosurgery in head and neck oncological and reconstructive surgery: personal experience on 127 cases. Acta Otorhinolaryngol Ital Organo Uff Della Soc Ital Otorinolaringol E Chir Cerv-facc 29: 1, 2009.

13. Schlee M, Steigmann M, Bratu E, Garg AK: Piezosurgery: basics and possibilities. Implant Dent 15: 334, 2006.

14. Landes CA, Stübinger S, Rieger J, Williger B, Ha TKL, Sader R: Critical evaluation of piezoelectric osteotomy in orthognathic surgery: operative technique, blood loss, time requirement, nerve and vessel integrity. J Oral Maxillofac Surg Off J Am Assoc Oral Maxillofac Surg 66: 657, 2008.

15. Yun KH, Kim SG, Lim SC: Comparison of New Bone Formation and Osteotomy Speed in the Rabbit Mandible Using Piezoelectric Surgery and Conventional Burs. Key Eng Mater, 2008. Available at: https://www.scientific.net/ KEM.361-363.1257. Accessed January 13, 2020.

16. Salami A, Mora R, Mora F, Guastini L, Salzano FA, Dellepiane M: Learning curve for Piezosurgery in well-trained otological surgeons. Otolaryngol--Head Neck Surg Off J Am Acad Otolaryngol-Head Neck Surg 142: 120, 2010.

17. Pappalardo S, Guarnieri R: Randomized clinical study comparing piezosurgery and conventional rotatory surgery in mandibular cyst enucleation. J Cranio-Maxillo-fac Surg Off Publ Eur Assoc Cranio-Maxillo-fac Surg 42: e80, 2014.

18. Melek LN, Noureldin MG: Comparative evaluation of piezotome versus periotome extractions of non-restorable endodontically treated teeth: A randomized clinical trial. Future Dent J, 2018.

19. Akçay H, Tatar B, Kuru K, Gözlüklü Ö, Ulu M: Bone Flap Technique With Piezosurgery for Impacted Teeth Extraction and Bone Cysts Removal Without Additional Fixation. J Craniofac Surg 30: e21, 2019.

20. Ihan Hren N, Miljavec M: Spontaneous bone healing of the large bone defects in the mandible. Int J Oral Maxillofac Surg 37: 1111, 2008.

21. Carini F, Saggese V, Porcaro G, Baldoni M: Piezolelectric surgery in dentistry: a review. Minerva Stomatol 63: 7, 2014.

22. Ibrahim YA, Aly TM, Ragab HR: A comparative study of mandibular cyst enucleation using the piezoelectric surgery versus the conventional technique. Alex Dent J 41: 350, 2016. 